\title{
Ecosystem Thinking and Methods in Design Morphology
}

\author{
Song Qiu ${ }^{*}$, Weizi $\mathrm{Xu}^{2}$, Xiao Zhang ${ }^{3}$, Qiang Cui ${ }^{3}$, Siyu Jin ${ }^{3}$ \\ 1* Room No.B439, Academy of Arts \& Design, Tsinghua University, Haidian District, Beijing 100084, China; \\ qiusongtty@sina.com; +86 13901179091 \\ 2, 3 Academy of Arts \& Design, Tsinghua University; \\ karen_xwz@163.com (W. X.); z-x20@mails.tsinghua.edu.cn (X. Z.); 542230410@qq.com (Q. C.); \\ lucky51king@163.com (S. J.)
}

\begin{abstract}
As an emerging discipline, Design Morphology, with the advantage of "Form Study", has been integrated with many disciplines, and gradually formed its collaborative innovation paradigm. The inclusion of "Ecology" into Design Morphology is expected to promote the research of Design Morphology with the help of the systematic thinking and methods of ecology. The ecosystem of Design Morphology includes the natural ecosystem and the quasi-ecosystem closely related to human beings, and also put forward the concept of "Form Community" for the first time in the design field. In fact, this is exactly the research scope of Design Morphology. Advocating the ecological view of Design Morphology, can not only help to design researchers improve their values and world view, with new thinking and method to engage in "Form Study", but also contribute to the theoretical construction and thinking expansion of Design Morphology, and play a positive role in promoting interdisciplinary collaborative innovation led by Design Morphology. In addition, it can be used to evaluate the overall research and future development trend of Design Morphology, and provide the new research ideas and approaches for the development of design.
\end{abstract}

Keywords: Design Morphology; Ecosystem Thinking and Methods; Ecological Environment; Form Community

Design Morphology [1] has always attached great importance to the core research work of "Form Study". However, in reality, any "Form" does not exist in isolation, but is closely related to its environment and the other forms, and even to human beings. Thus, "Form Study" is a systematic research with "Form" as the core, and each part of the system is not in a static state among each other, but always in a state of dynamic balance. Therefore, it is necessary to introduce the concept of "Ecology" in Design Morphology research in order to better carry out the systematic research centered on form.

\section{Design Morphology \& Ecosystem}

Ecosystem theory was founded by British plant ecologist Arthur George Tansley [2]. It is a dynamic equilibrium system composed of biocoenosis and its living environment. A biocoenosis is a group of animals, plants, and microorganisms that depend on each other in a natural environment. The biocoenosis continue to exchange material and energy flow with its living environment, and interact and influence each other to achieve the overall dynamic balance [3]. In fact, in addition to the natural ecosystem, there are also "Quasi-ecosystems" closely related to human beings. Although they are different from the natural ecosystem, they are closely related to each other. The artificial ecology refers to the "Artificial World" created or transformed by human beings with their own wisdom and hands, such as cities [4], communities, buildings, transportation, etc., which is a visible ecosystem intertwined with nature and sharing the benefits each other; The humanistic ecology refers to the "Humanistic World" formed and constructed by human beings with their cognition, emotion and consciousness, such as society, economy, culture and 
law, etc., which is an invisible ecosystem independent from nature. Both of the artificial ecology and the humanistic ecology are always interacting and influencing, and realizing dynamic balance under the leadership of human beings, that is the quasi-ecosystem.

In Design Morphology, its ecosystem will have a new perspective and interpretation due to the intervention of "Form Study". Ecosystems constructed from natural forms are still called "Natural Ecology", while those constructed from artificial forms are called "Artificial Ecology". Then, is there any relation between Design Morphology and "Humanistic Ecology" constructed by ideology? Of course! However, this correlation is reflected in the relationship between "Man" and "Form", not limited to the relationship between man and man. Thus, ecology in Design Morphology involves three factors: natural form, artificial form and human beings. They interact and depend on each other to form a complex and unified "Ecosystem" of Design Morphology. In spite of this, it is necessary to rely on the full support of science and humanities, if we want to deeply study the three parts and their mutual relations and make them coordinate with each other into a complete unity. Therefore, science and humanity constitute "Ecological Environment" of Design Morphology, while man and form constitute "Form Community" (analogous biocoenosis) of design morphology. In fact, the goal of Design Morphology's ecosystem is to achieve the dynamic balance between "Natural Ecosystem" and the "Quasi-ecosystems", by means of collaborative innovation with the help of scientific and humanistic ideas, and the core of which is to dynamically coordinate the relationships of natural form, artificial form and human beings [5]. Figure 1.

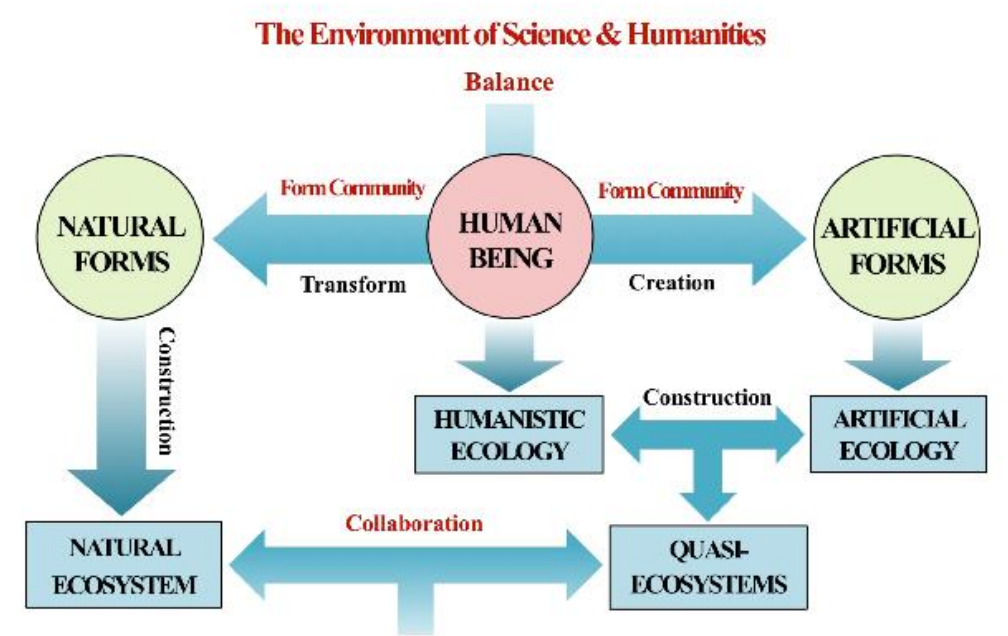

The Environment of Science \& Humanities

Figure 1. Ecosystem Construction of Design Morphology

\section{The Form Community of Design Morphology}

\subsection{The Role of Human Beings in the Form Community}

In the form community, human beings have certain dominance, but the premise is that human will and behavior must follow the law of natural ecology, otherwise it will backfire and cause ecological imbalance. In fact, in a broader sense, human beings are also a member of "Natural Form" and should be on equal terms with the natural form and the artificial form. Like other living things, humans will die and melt into the earth, mountains and rivers before returning to nature. So, what role should human beings play in the form community?

First of all, let us focus on the relationship between human beings and natural forms. There is no doubt that nature provides basic conditions and guarantee for human survival. However, while enjoying the gifts of nature, human beings are not satisfied with these. Therefore, human beings set off large-scale actions to transform nature, such as logging 
to build houses, clearing land for cultivation, domesticating livestock, and even building dams for water storage and irrigation. Although these are against the laws of nature, nature has been generous enough to accept them. If mankind had given up, it could have enjoyed its peace, however, if greedy enough, continue to destroy the ecology, resources, will inevitably lead to the punishment of nature. Thus, human beings and natural forms should be equal and reciprocal. Then, let's focus on relationship between human beings and artificial forms. Because artificial forms are created by humans, they are dominated by human will. However, human activities of creation are not unlimited, that is, never destroy the natural ecological balance, against human social ethics. To hold onto the upper limit, humans must constrain their actions and desires, and control the scale of artifacts (artificial forms). From this point of view, human beings and artificial forms are obviously a master-slave relationship, and the important role of human beings is to achieve the dynamic balance between artificial forms and natural forms [6].

Traditional design advocates the people-centered design [7], which puts human beings in the supreme position, so it is difficult to rely on human to balance the relationship between the artificial ecology and the natural ecology. The design morphology emphasizes "Form Study" as the core, which greatly reduces the importance of human beings, because human beings are actually a part of the nature forms. By treating humans and forms as equals, we can better clarify the true nature of the ecosystem of Design Morphology.

\subsection{The Core Function of the Form}

Form in design morphology can be understood from two aspects. On the one hand, form and matter can be said to be "Homogeneous but Different Performance". Both of them are essentially the same, but the perspective is different. Morphology attaches great importance to the relationship between appearance and essence, such as column, egg body, shell, etc. But, the matter emphasizes the relationship between properties and structure, for example metal, wood, cells, etc., are also collectively referred to as "Objective Forms". On the other hand, form can transcend the limitation of material and be closely connected with human consciousness, and create "Subjective Form" different from objective form from three aspects of cognition, emotion and will, such as geometric form, abstract form and illusory form. Thus, form is actually the unity of matter and consciousness. It can not only show the characteristics and performance of its objectivity (scientific nature), but also show the representation and intention of its subjectivity (humanistic nature).

Natural Form is the most common form in reality and also the leading role of natural ecology. There are many kinds of them and their composition is extremely complex. In order to facilitate the study, they are divided into two categories: living natural form (biological form) and inanimate natural form (abiotic form). The biological form is the form closest to human beings, and an indispensable friend of human beings. The biological morphology has its own inherent morphological characteristics, and according to the existing growth law of reproduction, growth and extinction. It is generally believed that the "Form" of living things is closely related to their habits and growing environment, and is constantly improved in the process of evolution. The abiotic form does not have a definite life span and constant growth and change posture like the biological form, but its existence forms are also kaleidoscopic and colorful, and can be transformed into each other under certain conditions. No matter what kind of form, even if the external influence is great, they will still strictly follow the internal law of life to grow and evolve [8].

Although nature is all-embracing and colorful, human beings are still not willing to be manipulated by it and resigned to fate. While constantly exploring and studying natural forms, they are also imitating and creating artificial forms different from nature. These forms are made with biological materials, artificial materials, and a variety of mixed materials. A large number of artificial forms gradually constitute a new ecosystem Artificial Ecology. As the protagonist of the artificial ecology, artificial form has gone through imitation, digestion, absorption, innovation and other stages. In fact, humans 
have been inventing since the dawn of time and continue to do so today. The innovative development of artificial forms also benefits from technological and design innovations. From the perspective of today's social development, scientific and technological innovation has played a key role in the development of human society, especially under the stimulation of advanced technologies such as artificial intelligence, quantum mechanics and synthetic biology, the quantity and quality of artificial forms have been unprecedented [9]. Figure 2.

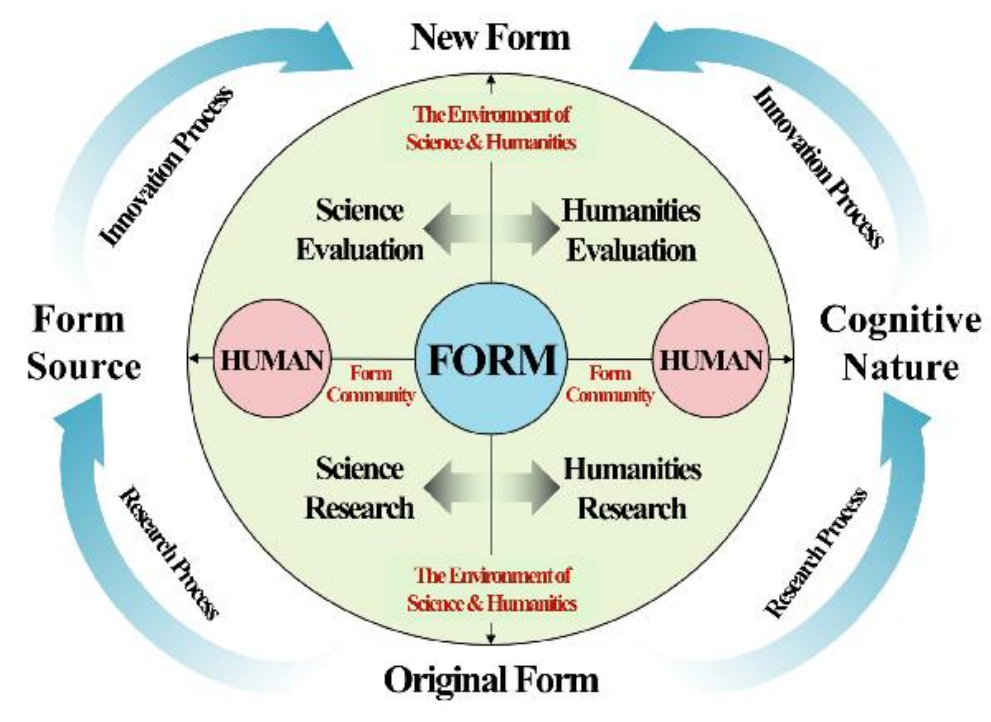

Figure 2. Form Communities and Ecological Environment of Design Morphology

\section{The Ecological Environment of Design Morphology}

Design Morphology, like other disciplines, has its own attributes. As its core is "Form Study", its attribute is bound to be closely related to "Morphological Attribute". In fact, form has a dual attribute, namely: objectivity (objective existence) and subjectivity (subjective perception). Only by recognizing and understanding form from two aspects can we grasp the whole picture and grasp the essence. Based on this, the basic attribute of Design Morphology is relatively clear, which is "Scientific" and "Humanistic". "Scientific Attribute" and "Humanistic Attribute" are two important attributes of design morphology. They interact and cooperate with each other to construct the ecological environment of Design Morphology.

\subsection{Scientific Attributes of Design Morphology}

The purpose of science is to understand reality through explanation. The unique (but not the only) method of criticism used by science is experimental verification [9]. The reason why Design Morphology strengthens its scientific attributes is to explore and study the laws, principles, essence and even origin behind the phenomenon of "Form" with scientific ideas and methods, and then create "New Form" through association and inspiration, and finally build the ecological system of Design Morphology. The research of Design Morphology is carried out around "Form", and form is the core component of "Form Community". If we want to uncover the inner mystery of form, we need the help of scientific thinking and methods, as well as the corresponding advanced technology to complete the assistance. Any form is the result of its internal and external causes. "Form" as the breakthrough point to study the formation of the internal cause, external cause multiple effects of combining form, through the observation, analysis, and hypothesis, and at the same time by using digital simulation and experimental verification method, finally confirmed the discovery, speculated that "Law", and then, after a lot of similar research in the form of validation, induces "Principle", and finally back to " Origin " of the 
form. In the process of the formation of the whole form, scientific thinking and methods always follow, playing an important role in protecting [10].

Design Morphology can also be understood as a process of "Deconstruction" of forms when exploring the laws and principles of natural forms. It is a working method from top to bottom, from the outside to the inside. Simple forms can be observed directly, while complex forms need to be observed with the help of advanced scientific equipment, and even need to learn the necessary pre-knowledge. In the stage of research and analysis, it is necessary to rely on the methods of experimental verification and simulation calculation to verify the reasoning and hypothesis of researchers, and then summarize the rules and principles of morphology. In the whole process, a lot of scientific knowledge and methods need to be applied, and some new knowledge and methods will also be produced. Under the research framework of Design Morphology, the research on the laws and principles of the construction of artificial form has many similarities with that of natural form, but there are also great conceptual differences. Exploring the rules and principles of the construction of artificial forms is more like the "Reconstruction" process of forms, which is a bottom-up, from the inside out working method. The construction of artificial form will focus on materials, structures and processing techniques closely related to the form. This means that it is more dependent on scientific knowledge and methods, because advanced technologies, new materials and new methods are the strong support behind it. In addition to learning from nature, the construction of artificial forms needs to be innovated and created, which promotes the integration of multiple disciplines, as well as the continuous updating of scientific and technological knowledge and methods [11].

In fact, whether exploring the unknown "Natural Form" or creating the future "Artificial Form", future science and technology is an important foundation and bridge to achieve their goals. For example, space science and technology determine the phenomenon and origin of human exploration of "Cosmographic Form"; Molecular biology leads the generation and construction of "Microscopic Form". Computer and brain science have made it possible for humans to cross the line between the real world (physical form) and the virtual world (virtual form). In the process of form exploration, research and innovation, researchers will gradually accumulate a large amount of practical experience. These experiences are then summarized, concluded and repeatedly verified to form a method for guiding practice. Then, after continuous improvement, optimization and systematization, the final formation of a complete theory. In the process from practical research to theoretical construction, scientific thinking and method also play a very important role, which is the important foundation and the beacon of Design Morphology theory and method construction [12].

\subsection{Humanistic Attributes of Design Morphology}

The humanistic attribute of Design Morphology corresponds to its scientific attribute, and it is also an important construction element of "Artificial Ecology". Because of human intervention, the objectivity of form is inevitably challenged, even the natural form is not immune to human interference, let alone human beings are members of the natural form. So, what are the humanistic attributes of Design Morphology? In fact, it is mainly embodied in two aspects: one is the relationship between man and form; the second is the cognitive law of form. The list of human relationships to form is endless, but it can be summed up in three broad categories: a) Primitive Form. Emphasize the intuitive feeling of human to the natural form; b) Intention Form. Emphasizing human cognition, transformation and innovation of natural form; c) Interactive Form. Emphasize the correlation and interaction between human and form (natural form, artificial form). These three forms are actually defined from the development degree or stage of the relationship between human beings and forms.

"Primitive Form" refers to the form that has not been transformed by human beings, which is actually the natural form. However, the difference between the two is that the primitive form has human participation, so the research content becomes complicated. At this stage, however, human beings are only mildly involved by intuition. For example, 
bathing in clear hot spring, feel soothing and relaxed; hearing the birds 'song and smelling fragrance of flowers, immediately relaxed and happy....... These feelings are the result of unthinking human intuition and nature. It does not lead to great differences in intuition between individual humans, and therefore is more cognitively universal when exploring unknown forms. It can be seen that the study of primitive form naturally becomes an important research basis for the humanistic attributes of Design Morphology [13].

Compared with the Primitive form, the "Intentional Form" is the close relationship formed by the deep participation of human beings, which is not only reflected in human's subjective cognition of form, but also reflected in human's transformation and innovation of form. Due to the individual differences of human beings, even the subjective cognition of the same form will be greatly different. This adds further complexity to the study of form cognition, but it also makes intentional forms richer and more varied. It should be emphasized that human cognition of form only stays at the level of consciousness, without really touching form. The transformation and innovation aimed at form is completely different. They will not only touch form, but also transform form, and even create new form. In this way, we enter into the research category of artificial form, that is to say, we step into the "Artificial Ecology" constructed according to human will [14].

As for "Interactive Form", it develops on the basis of the cognition of the first two forms. The relationship between man and form is not only one-way development, in fact, more cases exist in the form of "Interaction", such as touching the leaf of mimosa, the leaf will quickly fold up; When you bend the wood, its elasticity squeezes your hands. Aquarium dolphins, according to the trainer's instructions to complete a variety of movements; AI robots can conduct dialogue and body communication with human beings. Humans can also perceive and manipulate virtual forms in virtual reality and so on. "Interactive Form" not only focuses on the role and effectiveness of human beings in giving forms, but also attaches great importance to the timely feedback of forms. In addition, innovation based on the relationship between the two may build a new ecological environment. Compared with the first two forms of cognition, interactive form strengthens the closeness and balance of the relationship between human and form, adds more uncertainty, and brings more opportunities and changes for design innovation.

The study of the humanistic attributes of Design Morphology is different from the reflection on human beings in philosophy, and different from the sociological classification of human beings, also different from the psychological analysis of human beings. The study of humanistic attribute focuses on the exploration of human vitality, and the communion of man with all things, and summarizing the sources of human creativity. It may be too cutting-edge to be understood and applied, but its breakthrough research method will have important reference value for future design and even interdisciplinary collaborative innovation [15].

\section{Case Studies of Ecosystems Thinking and Methods in Design Morphological}

\subsection{Design Research of Thermoelectric Power Generation Products Based on the Ecological Environment of Herdsmen in Inner Mongolia}

In Inner Mongolia, herdsmen often live on nomadism. Nomadism is an agricultural production activity that is very friendly to the ecological environment. In the process of nomadism, herdsmen drive their animals to different pastures to avoid excessive consumption of vegetation. At the same time, animal manure also provides nourishment for large pastures. Livestock will become the daily food supplement for herdsmen, and more will be traded and become an important source of income for herdsmen. Thus, nomadism has become a way of life in which herdsmen and nature coexist harmoniously. However, with the development of modern society and economy, electricity demand has become a new important demand in herdsmen's life [16].

However, since herdsmen often need to nomadize on the vast grassland, it is difficult for fixed transmission lines to serve them effectively. Herdsmen usually use solar and wind energy to obtain electricity. In addition, due to the shorter sunshine time in winter, 
solar energy often cannot provide enough power. Therefore, an idea comes to us: whether we can provide herdsmen with electricity except for wind and solar by burning the dung of grassland livestock. During the field investigation period, The research team went to Inner Mongolia for more detailed living status and surrounding ecological environment. In this survey, we visited four herdsmen's homes in the depths of semi-desertification grassland. As the pastoral area is located at high latitude, herdsmen need to make a fire for heating for more than five months of the year, which is very consistent with the characteristics of thermoelectric power generation. Figure 3.
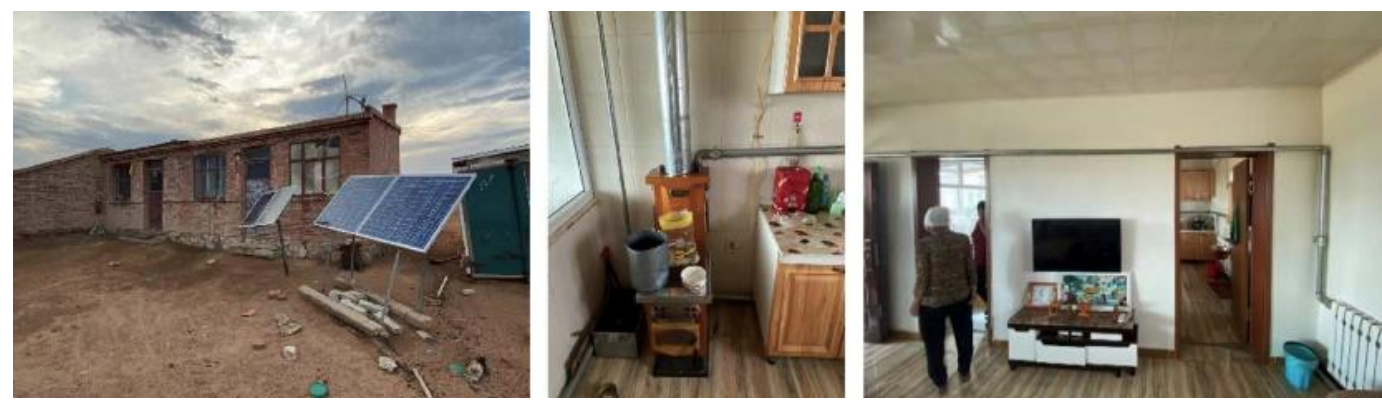

Figure 3. Herdsmen's living power generation equipment, stoves and heating equipment

Thermoelectric power generation is the mutual conversion of thermal energy and electric energy realized by thermoelectric effect of thermoelectric power generation materials, produced by temperature difference. In the traditional thermoelectric power generation equipment (TPGE), most of the equipment adopts air cooling to maintain the temperature difference and support the combustion of the stove at the same time [17]. However, Hot air is hard to store heat effectively. So, the research team decided to change the original thermoelectric refrigeration mode from air cooling to water cooling. In thermoelectric power generation, cooling water with waste heat can be used as heat storage medium for the whole house in winter. At the same time, researchers found that water cooling can improve the efficiency of large temperature difference power generation more than air cooling. Which means under the same external conditions, the efficiency of water-cooled power generation is more than three times that of air-cooled power generation. Moreover, due to the good heat storage performance of water, even if the hot end is no longer heating, warm water can continue to generate electricity for more than 2 hours [18]. Figure 4.

Electric power is the living necessity and economic development guarantee of human society. According to the research released by the International Energy Agency, it is estimated that more than 700 million people will still have no access to electricity by 2040, accounting for $10 \%$ of the world's total population. In China, there are still about 28000 villages, 7 million households and more than 30 million people in rural and pastoral areas that need to be solved urgently. Therefore, the research of independent power generation equipment combined with ecological environment is always a subject we have to face. Herdsmen burn livestock manure to obtain heat for heating in winter, and use thermoelectric power generation technology to supplement the power needed in winter. The burned animal manure can be used as fertilizer to nourish the grassland for livestock consumption. Thermoelectric power generation technology is a way to convert low-level energy (internal energy) into high-level energy (electric energy). At the same time, thermoelectric power generation equipment (TPGE) is keeping herdsmen's lives in the system of grassland ecological cycle. Our design and research is not to transmit electricity and heat to herdsmen, but a natural energy conversion device, so that herdsmen can spontaneously convert the "waste" in the ecological environment into energy in their nomadic life on the grassland [19]. Figure4. $(\mathbf{a}, \mathbf{b})$ 


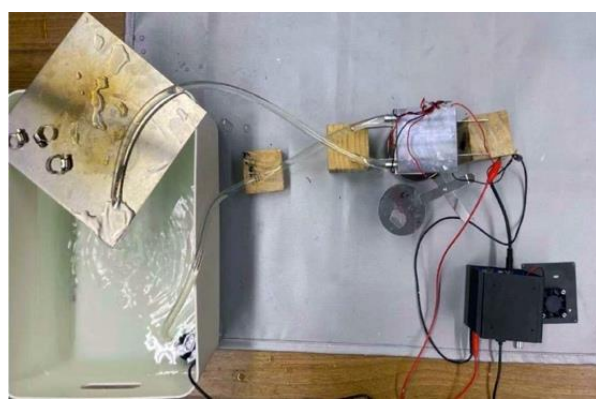

(a)

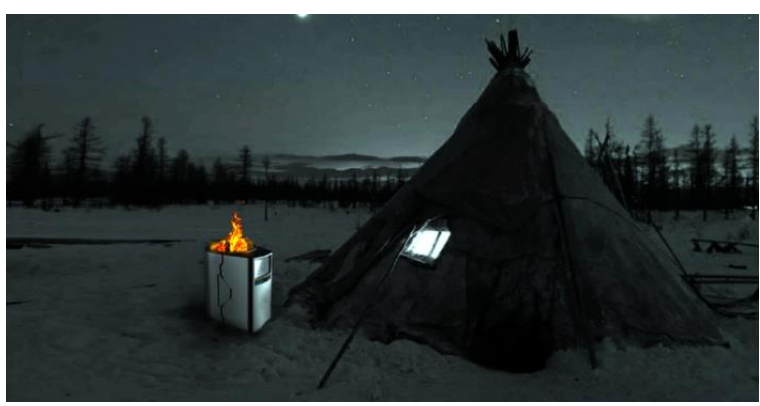

(b)

Figure 4. (a) Prototype of the water-cooled TPGE; (b) Application scenario of TPGE.

\subsection{Research on Intelligent Product Design Based on Digital Ecosystem}

A typical example of a dynamic equilibrium system in nature is slime molds. Slime molds are highly efficient at exploring their environment and achieving dynamic balance through its interaction with the environment. As slime molds search for food, they spread out, forming a dense network. When food is found, the useless branches die off, leaving only the best route to the food. In this way, they save energy and get the most food in different environments. The way slime molds find the best way to connect to food is often used to solve real-world path-planning problems in artificial ecosystem. The study on path planning of slime molds began with a maze experiment. It was then applied to simulate railway design in Tokyo. Engineers have spent more than 100 years optimizing the complex railway system, but slime molds have achieved similar results in just 26 hours. Figure 5.

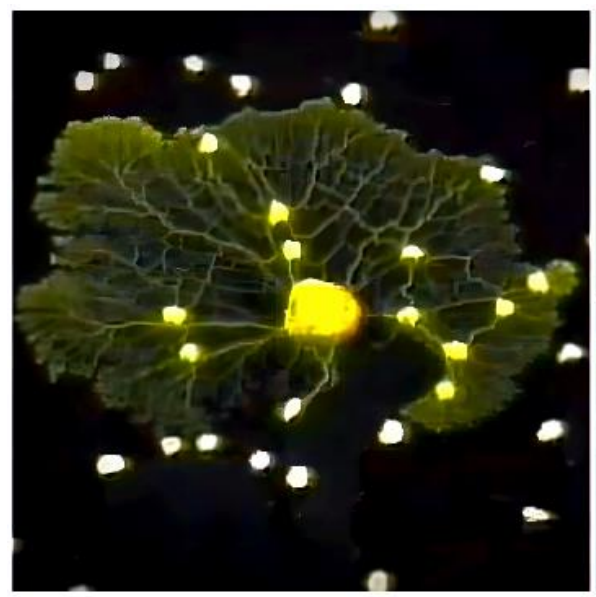

(a)

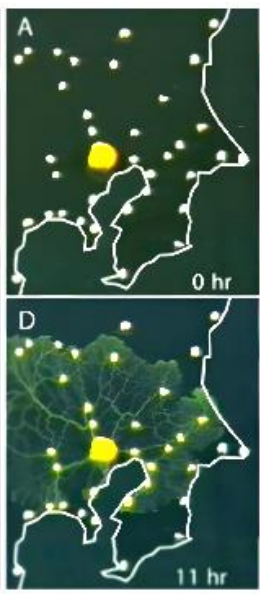

11

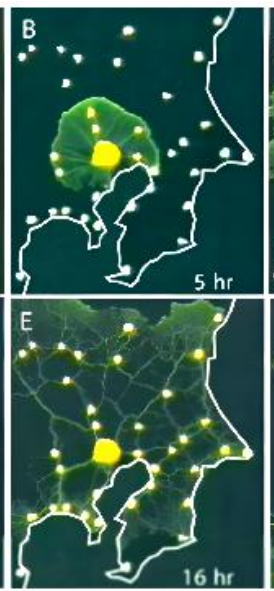

(b)

Figure 5. (a) The foraging behavior of slime molds; (b) the path planning experiments [20].

Based on the inspiration of slime mold foraging behavior, we designed a customized electric vehicle chassis. Chassis is the most basic part of electric vehicle, it is affected by the external environment, such as the load situation in different environments. In addition, the lightweight chassis has an obvious effect on reducing energy consumption. So, the chassis needs to meet the requirements of strength and lightweight. If the structural optimization process of electric vehicle chassis is abstracted into connecting a group of points with a certain amount of lines [21], the slime mold algorithm is also applicable to the design of chassis.

The slime mold algorithm first needs to determine the number and location of key points. Just like the slime mold, all the connecting lines will be calculated. Each point is then randomly assigned a weight value, then the structural path is generated at the point with the highest weight. When a connection line is generated at the point with the highest weight, the weight of that point is reduced to give other points an opportunity. Finally, 
genetic algorithm is used to optimize and adjust the weight distribution, so as to create the next generation of better structure.

For chassis design, a set of points are defined in the space according to the layout of the main components, which are similar to the food source of slime molds. After obtaining the structural lines of the chassis through the slime mold algorithm, finite element analysis (FEA) was performed based on the objective of maximum deformation and stress. This measurement allows us to set optimization problems where reducing the mass of the structure is the goal and performance measurements are used as constraints. For example, the constraint could be that the maximum deformation of the structure cannot exceed $5 \mathrm{~mm}$. After the optimization model is determined, the genetic algorithm is used to search the solution in the design space, and the result is visualized. As you can see from the Figure, the algorithm creates more branches where the load is highest, which emphasizes the effectiveness of ecological design morphology [22]. Figure 6.
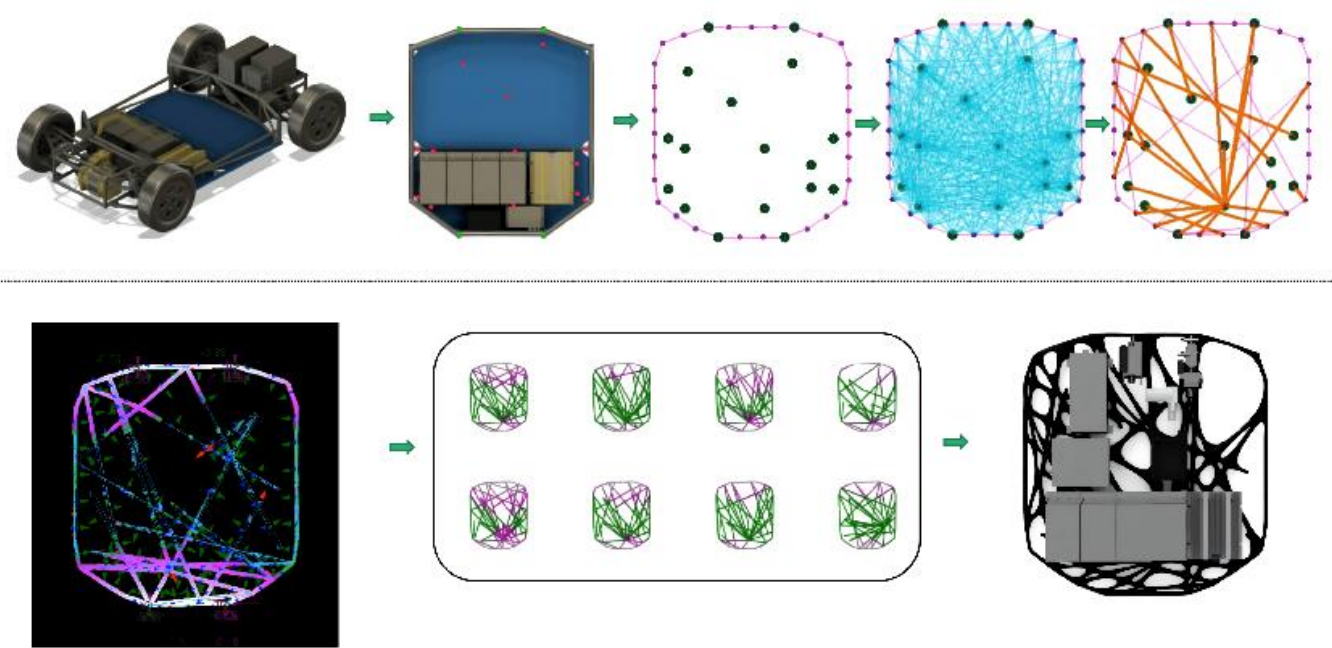

Figure 6. The design process of a customized electric vehicle chassis by the slime mold algorithm

\section{Conclusion}

Incorporating the concept of "Ecosystem" into Design Morphology is actually to study Design Morphology with the help of the system thinking of "Ecology". The ecosystem of Design Morphology includes not only the natural ecosystem, but also the quasi-ecosystem closely related to human beings. In fact, this is the research scope of Design Morphology [23]. Thus, the research scope of Design Morphology is broader, more complex and more variable than that of ecology. Therefore, in order to study Design Morphology deeply and comprehensively, we should not only study unilateral factors in a single "Form Community", but must integrate all factors in all form community into the ecological environment of Design Morphology.

In the natural ecosystem, on the one hand, human beings need to follow the laws and basic principles of the natural form while relying on science and technology to explore and transform the natural form [24]; On the other hand, it is necessary for human beings to constantly learn and gain enlightenment through the study of natural forms with the help of humanistic knowledge, so as to promote and enhance their creativity [25]. In the quasi-ecosystem, human beings not only rely on science, technology and humanistic knowledge to create artificial forms, but also need to constantly reflect on their behavior with the help of humanistic thoughts and criticism while enjoying artificial forms. Therefore, Design Morphology should not only pay attention to the dynamic balance between human being, natural forms and artificial forms, but also strengthen the dynamic balance between natural ecosystem and quasi-ecosystem. To ensure its dynamic balance, we must rely on the ecological environment of science and humanities. Although the ecological balance is the goal pursued by human beings, its equilibrium state is not lasting, even just a moment [26]. Figure 7. As Kevin Kelly points out in Out of Control, "A system 
that is not stable at a certain equilibrium point is almost equivalent to an explosion and is bound to die quickly. Nothing can be both in equilibrium and out of balance. But things can be in a permanent disequilibrium -- as if surfing on an edge that never stops and never falls. The magic of creation is to settle down at this critical point of flow, which is what mankind has been striving for." [27]

As the ecological environment of Design Morphology, science, technology and humanities also achieve their dynamic balance in the competition. The highest goal that the mind of man has ever tried to achieve is to combine science and the humanities [28]. As a matter of fact, scientific and technological progress is a double-edged sword. While benefiting mankind, it is just like a runaway horse constantly breaking the ecological balance that human beings are accustomed to. Humanistic thought is just like a horse trainer constantly restraining and revising its behavior to bring its into the right track of benign ecological development. Advocating ecological design morphology, but also can improve design researchers values and world view, to help them with new thinking and perspective of "Form Study", at the same time, also for Design Morphology has a powerful role in promoting the theory of construction, design and the morphology of interdisciplinary collaborative innovation have enlightenment role, coordination and balance. In addition, it can also comprehensively evaluate the overall research ability and future development trend of design morphology, and provide a good research idea and new paradigm for the development of design science. Figure 8. $(\mathbf{a}, \mathbf{b}, \mathbf{c})$

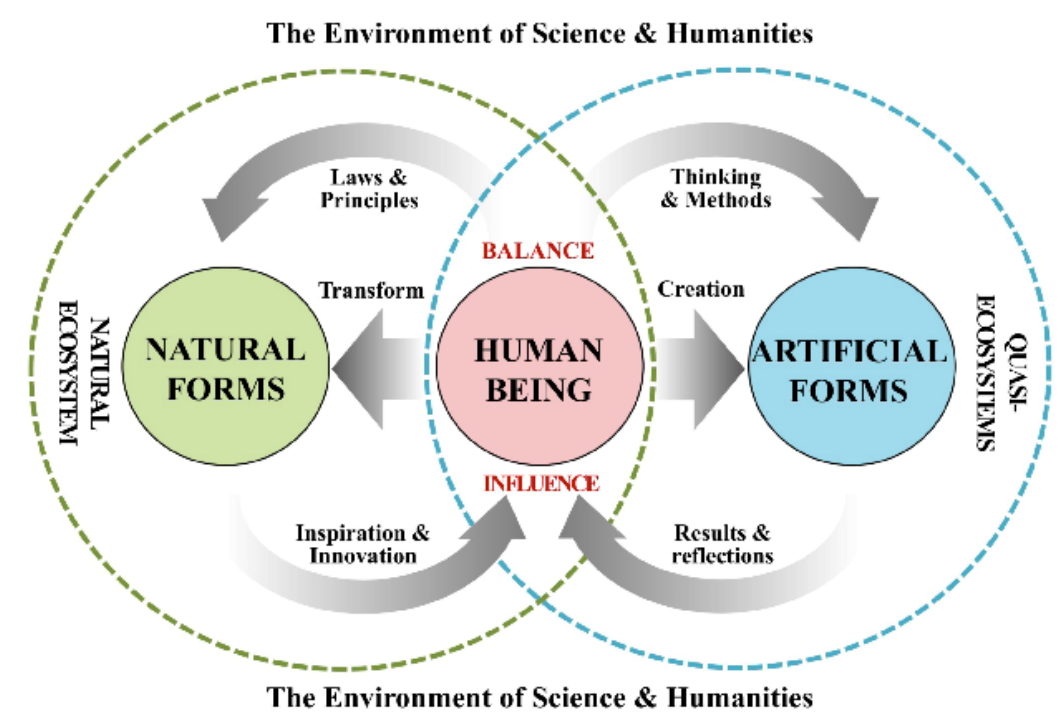

Figure 7. Design the Dynamic Cycle Pattern of the Ecosystem of Design Morphology

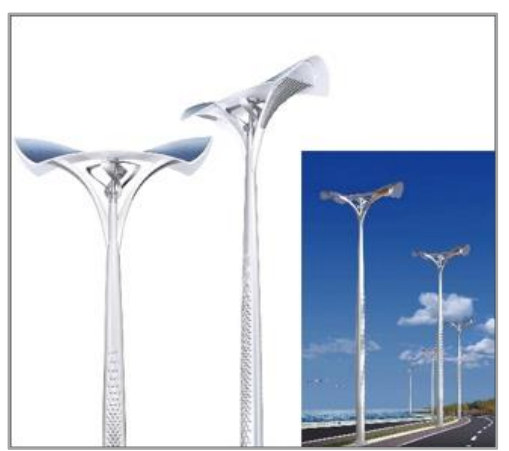

(a)

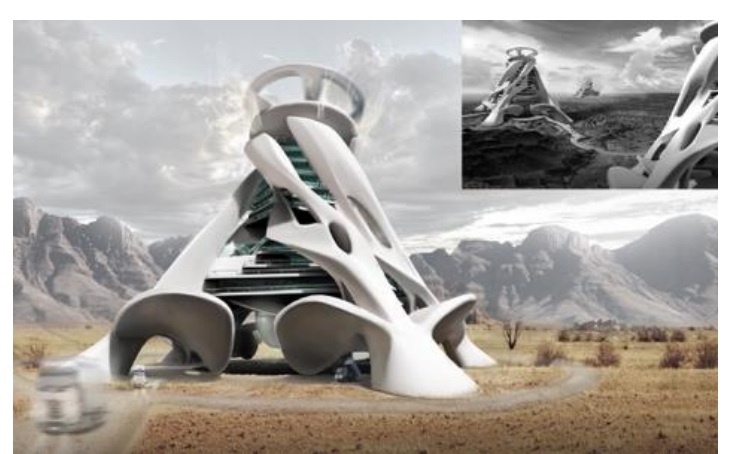

(b)

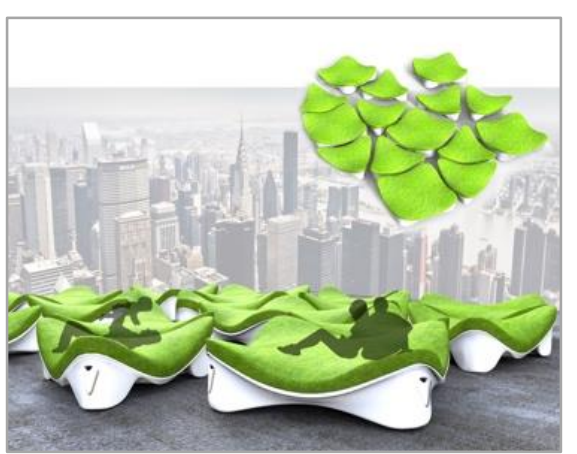

(c)

Figure 8. (a) Breathing lamp (designed by Qiu Song and Bai Ying): Wind energy and solar energy complement each other to provide clean energy for street lamps, and realize efficient lighting of urban roads and purification of automobile exhaust; (b) Super Beetle (by Song Qiu and Yacheng Han): The municipal waste is processed into harmless mud by "super beetle" and injected into abandoned mines to reduce the occurrence of secondary disasters in "mined-out areas"; (c) Green 
City Furniture (designed by Qiu Song and Han Yacheng): Movable and spliced grass furniture is placed in densely populated areas of the city, and the grass is cultivated without water, allowing people to get close to the long-lost nature in leisure time.

Author Contributions: Conceptualization, S. Q, and W. X.; Methodology, S. Q.; Software, Q. C.; Validation, X. Z. and Q. C.; Formal Analysis, W. X. and S. J.; Investigation, X. Z. and Q. C.; Resources, S. J. X. Z. and W.X.; Data Curation, S. J.; Writing-Original Draft Preparation, W. X. and S. J.; Writing-Review and Editing, S. Q. and W. X.; Visualization, S. Q.; W. X.; X. Z. and Q. C.; Supervision, S. Q.; Project Administration, S. Q. and S. J.; Funding Acquisition, NSSFC. All authors have read and agreed to the published version of the manuscript.

Funding: This research was funded by NSSFC, China, grant number 17ZDA019. https://xm.npopsscn.gov.cn

Data Availability Statement: Not applicable.

Acknowledgments: We would like to thank NSSFC for supporting this research, and thank Tsinghua University for providing many administrative and technical support, and also thank all authors very much for making a great contribution to the research.

Conflicts of Interest: The authors declare no conflict of interest.

\section{References:}

1. Voyatzakim, Gourdoukis D. From Morphology to Morphogenesis: On Speculative Architectural Design Pedagogy, in Handbook of Research on Form and Morphogenesis in Modern Architectural Contexts. IGI Global. 2018; pp. 20-40.

2. Editorial Department of Encyclopedia of China. Environmental Science, in Encyclopedia of China, Encyclopedia of China Press, China, 2010.

3. Eugene P. Odum; Gary W. Barrett. Fundamentals of Ecology, Higher Education Press, China, 2009; pp. 15-20.

4. Kongjian Yu; Dihua Li; Qingping Ji. Landscape \& Urban Ecological Design: Concept and Principle, Chinese Landscape Architecture, 2021, 06.

5. Li Bo. ECOLOGY, Higher Education Press, China, 2000; pp. 113-115.

6. $\quad$ Edward O. Wilson. Consilience: The Unity of Knowledge, CITIC Press, China, 2016; pp. 375-417.

7. Shengxi Fan, Human-centered Design from the Views of Behavior and Cognition. Journal of Machine Design, China, 2013, 30(2), pp. 97-99.

8. D'Arcy Thompson. On Growth and Form, Shanghai Scientific \& Technical Press, China, 2023; pp. 299-316.

9. David Deutsch. Context of the Real World: Parallel Universes and Implications, Posts and Telecommunications Press, China, 2016; pp. 69-71.

10. A.F. Chalmers. What is This Thing Called Science, The Commercial Press, China, 2016; pp. 57-84.

11. Wayne Weiten. Psychology Themes and Variations, China Machine Press, China, 2023; pp. 115-117.

12. Chinese Society of Astronautics. 2049 Vision of China's Science and Technology and Society: Space Science and Technology and China's Sky Dream, Science and Technology of China Press, China, 2020; pp. 109-120.

13. James J.Gibson. The Ecological Approach to Visual Perception. Psychology Press, China; 1986.

14. Masayuki Kurokawa. Design Rhetoric, Hebei Fine Arts Publisher, China, 2014; pp. 46-50.

15. Jun Zhao. Cross-cultural Communication: Cognitive Differences and Cultural Language Acquisition, Beijing University Press, China; 2012.

16. Li Yanzu. Beauty of Creation: Art and Culture of Product Design. Renmin University Press, China; 2000.

17. Jianyun Zhao; Dongsheng Zhu; Zeguang,Zhou; Changhong Wang; Hong Chen. Research Progress and Present Situation of Thermoelectric Power Generation Technology. Power sources technology 2010, 34 (03), pp. 310-313.

18. Teng Zhang; Zheng Zhang. Temperature Difference Power Generation Technology and some Applications. Energy technology 2009, 30 (01), pp. 35-39.

19. Beijing University of Technology, Zuoren Nie; Zhihong Wang. Eco-environmental Materials Science. China Machine Press, China; 2004.

20. Tero A; Takagi S; Saigusa T; et al. Rules for biologically inspired adaptive network design, SCIENCE, 2010,327 (5964), pp. 439-442.

21. Kondo Shigeru; Miura Takashi. Reaction-diffusion Model as a Framework for Understanding Biological Pattern Formation. SCIENCE, 2010, 329 (5999).

22. Mu Li. Preliminary Study on Application of Automobile Modeling Design based on Parametric Design. Art science and technology, 2013, 26 (04), pp. 198.

23. Song Qiu et al. Research and Application of Design Morphology, China Architecture and Architecture Press, China; $2019 ;$ pp. 1-5.

24. Goss Julie. Biomimicry: Looking to nature for design solutions. Masters Abstracts International, 2009.

25. Zaiguang Zhao. Cultural Wisdom of the Unity of Nature and Man -- Chinese traditional Ecological culture and philosophy. Culture 
and Art Publisher, China, 2006; pp. 2-19.

26. Takeshi Goto; Massato Sasaki; Naoto Fukasawa. The Ecological Approach to Design. Guangxi Normal University Press, 2016; pp. 25-33.

27. Kevin Kelly. Out of Control, New Star Press, China, 2010; pp. 694-695.

28. Edward O. Wilson. Consilience: The Unity of Knowledge, CITIC Press, China, 2016; pp. 13. 